\title{
An efficient wireless network discovery method for vertical handover between WiMAX and WLAN
}

\begin{abstract}
One of the most difficult tasks for coordination the vertical handover is the discovering currently available radio access networks. Although the mobile nodes (MN) is easily can access to Worldwide Interoperability for Microwave Access (WiMAX) network, but continually it would have to discover available wireless local area network (WLAN) networks, which provide high data rates but have limited coverage area. This process discovers has a significant effect on the discovery time and power consumption for MNs. this article introduce a new technique to enhance access router discovery (EARD) method to solve this problems. Our proposal EARD method, previous access router provides information about the neighbouring networks for $\mathrm{MN}$ to discover available networks as soon as possible. We can see through simulation results that by enabling the EARD method has improved power consumption and the discover time of networks in the performance of MNs compared to the conventional network discovery techniques.
\end{abstract}

Keyword: Wireless; Computer network; Handover 